\title{
An Overview of IEEE Standardization Efforts for Cognitive Radio Networks
}

\author{
Ahmed Khattab \\ Electronics and Electrical Communications Department \\ Cairo University \\ Giza, Egypt \\ akhattab@ieee.org
}

\author{
Magdy A. Bayoumi \\ The Center for Advanced Computer Studies (CACS) \\ University of Louisiana at Lafayette \\ Lafayette, LA \\ mab@cacs.louisiana.edu
}

\begin{abstract}
The Cognitive Radio (CR) technology is envisioned as a primary solution for the spectrum scarcity problem in wireless communication networks. Cognitive radio networking allows the exploitation of the unutilized spectrum bands in an opportunistic manner, and thereby, improves the efficiency of the spectrum utilization. Cognitive radio Networks (CRNs) provide wireless connectivity via heterogeneous wireless architectures and dynamic spectrum access techniques. In this paper, we present an overview of the state-of-the-art of the IEEE standardization efforts for CR and CRNs.
\end{abstract}

Keywords-cognitive radio networks; TV white spaces; IEEE 802.11af; IEEE 802.22; IEEE 1900

\section{INTRODUCTION}

Cognitive radio networking has recently presented itself as a key technology to alleviate the severe spectrum underutilization and provide a solution for spectrum scarcity [1]. Cognitive Radio Networks (CRNs) are foreseen as a fundamental component in future wireless Information and Communications Technologies (ICT) that exploit dynamic and opportunistic spectrum access strategies to provide wireless connectivity [2]. The enabling component of CRNs and opportunistic spectrum access is the cognitive radio $(\mathrm{CR})$. A CR is a wireless device that senses the surrounding radio environment and opportunistically accesses the unutilized spectrum bands based on the activities of the surrounding primary licensed networks [3]. Intensive research and industry efforts have been carried out on the different ideas and architectures of CRNs over the past decade [1]. Therefore, standardization is needed in order to facilitate the development of the various ideas related to CRNs into commercial products.

Many standards have recently emerged to address the architectures, the operation, and/or the physical (PHY) layer and Medium Access Control (MAC) layer designs of CRNs such as the IEEE 802.11af, IEEE 802.19, IEEE 802.22, 3GPP ANDSF, and ECMA 392 standards. In this paper, we spot the light on the IEEE standards for cognitive radio networks. We first discuss the IEEE 802.22 and IEEE 802.11af standards. A common feature of the IEEE 802.22 and IEEE 802.11af standards is that they both operate in the TV White Space (TVWS) band. The propagation characteristics of the TVWS band make it a desirable and convenient spectrum for many wireless transmission services. More specifically, the material obstruction in the TVWS band is less harmful and the low operating frequency of TVWS significantly reduces the path loss compared to the unlicensed industrial, scientific, and medical (ISM) bands ( 2.4 and $5.7 \mathrm{GHz}$ ). We also overview the IEEE 1900 Dynamic Spectrum Access Networks (DySPAN) standard which also includes Working Groups (WGs) that target the operation over the TVWS bands such as the IEEE 1900.4 and the IEEE 1900.7. However, the main goal of the DySPAN standard is to standardize the definitions, procedures, and the interoperability rules of CRNs. ${ }^{1}$

\section{IEEE 802.22 STANDARD: WIRELESS REGIONAL AREA NETWORKS (WRANS)}

Despite the recent growth in the wireless communications industry, there still exist large unexploited markets for broadband wireless access in unserved/underserved areas where the wired infrastructure is economically not feasible to deploy. Thus motivated, the IEEE 802.22 standard: "Wireless Regional Area Networks (WRANs): Enabling Rural Broadband Wireless Access Using Cognitive Radio Technology in TV Whitespaces" was initiated to provide reliable and secure broadband wireless connectivity to underserved and unserved communities. TV white spaces are the unused spectrum resources at specific times and locations in the broadcast TV operating frequencies known as the VHF/UHF band that can be exploited through spectrum sharing. In the United States, the abandoned television frequencies are primarily in the upper UHF $700 \mathrm{MHz}$ band, covering TV channels 52 to 69 (698 to $806 \mathrm{MHz})$. Other non-continuous vacant bands between $54 \mathrm{MHz}$ and 698 $\mathrm{MHz}$ are also available. In other countries worldwide, the abandoned television channels are in the VHF band as the case in Europe where TV broadcast rang from $470 \mathrm{MHz}$ to $790 \mathrm{MHz}$. The TVWS band is still used by several licensed protected services such as terrestrial TV broadcast services and program making and special event (PMSE) services that have been operating over these bands for the past century. Being officially licensed, the incumbent users of the TVWS bands are protected from interference within their service area. In order to allow cognitive radio devices to operate in the TVWS band, they must not interfere with the protected incumbent users in their specified operating areas.

The IEEE 802.22 incorporates advanced cognitive radio capabilities including dynamic spectrum access, incumbent database access, accurate geolocation techniques, spectrum sensing, regulatory domain dependent policies, spectrum etiquette, and coexistence to increase the efficiency of spectrum utilization in spectrum currently allocated to, but unused by, the TV broadcast service. The IEEE 802.22 standard takes advantage of the advantageous communications features of the VHF and UHF TV bands to provide up to $22 \mathrm{Mbps}$

\footnotetext{
${ }^{1}$ The IEEE 802.19 Technical Advisory Group (TAG) is currently addressing the coexistence between wireless standards under development within the IEEE 802 [4]. The IEEE 802.19 is still in its early development phases. We do not further discuss this standard.
} 
broadband wireless access over a cell radius of up to $100 \mathrm{~km}$ (if power is not an issue $)^{2}$ without interfering with the reception of existing TV broadcast stations, using the so-called white spaces between the occupied TV channels. While the initial version of the standard does not allow mobility, the physical layer of the IEEE 802.22 standard can support mobility of up to 114 kilometer per hour. The IEEE 802.22 Working Group (WG) was initiated as the first worldwide effort to develop a cognitive radio-based air interface (i.e., the PHY and MAC layer specifications) in November 2004 and the standard was published in July 2011 [5]. Several amendments and subsequent versions of the standard have been released since then including the IEEE 802.22.1, IEEE 802.22.2, IEEE 802.22a and IEEE 802.22b standards [5].

The IEEE 802.22 system specifies a fixed point-tomultipoint wireless air interface whereby a Base Station (BS) manages all of the associated Consumer Premise Equipments (CPEs) within the cell. The centralized BS is responsible for the spectrum access decision and the transmission parameters (e.g., modulation, coding, and frequencies of operation) for all of the associated CPEs in both the downlink and uplink. Each BS is equipped with a Global Positioning System (GPS) receiver which would allow its position to be reported. The location information would be then used to obtain the information about available TV channels through a central server. The IEEE 802.22 standard adopts a strict master/slave relationship in which the BS acts as the master and the CPEs are the slaves. Such a master/slave approach ensures the protection of incumbent services such as the TV service and wireless microphones. The CPEs are not allowed to transmit before receiving the proper authorization from a BS.

In order to improve the accuracy of spectrum sensing and ensure proper incumbent protection, the IEEE 802.22 BS exploits distributed sensing which have various CPEs performing distributed measurement activities. A BS distributes the sensing load among the CPEs within its service area. Based on the received information, the BS better decides the best course of action to be taken.

\section{IEEE 802.11AF STANDARD: IEEE STANDARD FOR TV WHITE SPACES OPERATION}

The IEEE 802.11af standard, released in June 2013 and approved in February 2014, defines the modifications to both the 802.11 physical (PHY) layer and the 802.11 Medium Access Control (MAC) layer, to meet the legal requirements for channel access and coexistence in the TVWS bands between $54 \mathrm{MHz}$ and $790 \mathrm{MHz}$ [7]. Similar to the IEEE 802.22 WRAN standard, the IEEE 802.11af standard allows white space devices (WSDs) to share the underutilized TVWSs in many geographical locations when the primary incumbent services (such as licensed TV broadcasters and PMSE services) are not using such bands. The IEEE 802.11af standard guarantees the protection of incumbent users of the TVWS band from interference in their operating regions. The IEEE 802.11af aims at exploiting the superior propagation quality of the TVWS bands to achieve high performance. Due to the difference in TVWS bands worldwide, the IEEE 802.11af standard provides an international framework that adapts to the different TVWS operating parameters and regulatory domains around the world. Thus, the IEEE 802.11af supports different channel bandwidths which are learnt from an approved geolocation database that

\footnotetext{
${ }^{2}$ The current specified coverage range is $33 \mathrm{Km}$ at 4 Watts CPE effective isotropically radiated power (EIRP).
}

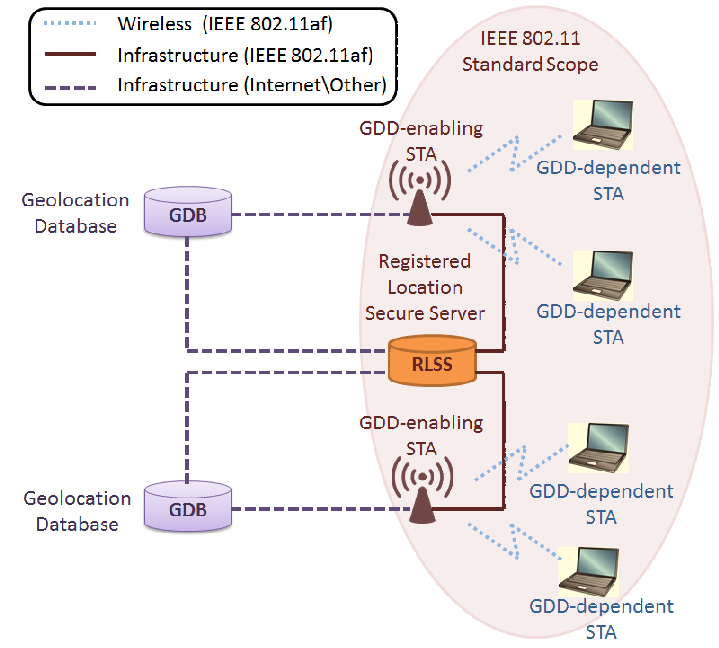

Fig. 1. General architecture of a typical IEEE 802.11af network [7], [8].

keeps track of which channels are available and for how long. The WSDs immediately cease transmission when the database informs them to stop in order to not interfere with the incumbent users. The IEEE 802.11af standard is commercially known as "Super Wi-Fi" or "White-Fi".

Figure 1 depicts the main components of a typical IEEE 802.11 af architecture which are:

- Geolocation Database (GDB): This database stores the permissible frequencies and operating parameters to fulfill the regulatory requirements for different geographic locations with accuracy 50 meters as specified by the standard.

- $\quad$ Registered Location Secure Server (RLSS): This server acts as a local database that contains the geographic location and operating parameters for a small number of basic service sets (BSSs). The operation of the access points (APs) and stations (STAs) in a given BSS is based on the RLSS information.

- Geolocation-Database-Dependent Enabling Station (GDD-Enabling STA): This component corresponds to the AP of legacy IEEE 802.11 standards. The GDD-enabling STA controls the operation of the STAs within its BSS service area. The GDD-enabling STA gets the available channel information from the GDB, and transmits the contact verification signal (CVS). The CVS is used to verify that the GDD-dependent STAs are still within range of GDD-enabling STAs, as well as for checking the list of available bands.

- Geolocation-Database-Dependent Dependent Station (GDD-Dependent STA): A GDD-dependent STA uses the available TVWS channels under the control of the serving GDD-enabling STA. The GDDdependent STAs obtain a White Space Map (WSM) from either the GDD-enabling STA or the RLSS that identifies the permissible operating frequencies and regulatory parameters in the operating area. The used communications protocol is called the Registered Location Query Protocol (RLQP). The GDDdependent STAs are equivalent to the STAs in the BSS architecture of legacy IEEE 802.11 standards. 
TABLE I. COMPARISON OF THE IEEE 802.22 AND IEEE $802.11 \mathrm{AF}$ STANDARDS

\begin{tabular}{|c|c|c|}
\hline & IEEE 802.22 & IEEE 802.11af \\
\hline \hline Coverage Range & $17-33 \mathrm{~km}$ (up to $100 \mathrm{~km})$ & $100 \mathrm{~m}-1 \mathrm{~km}$ \\
\hline Max. PHY rate & $22 \mathrm{Mbps}$ & $12 \mathrm{Mbps}$ \\
\hline Power & $4 \mathrm{~W}(36 \mathrm{dBm})$ & $100 \mathrm{~mW}(20 \mathrm{dBm})$ \\
\hline Sensitivity & $-97 \mathrm{dBm}$ & $-64 \mathrm{dBm}$ \\
\hline MAC & TDMA & CSMA \\
\hline Topology Support & Cellular & Mesh \\
\hline Mobility & Supported & Not Supported \\
\hline Geolocation Devices & Used & Used \\
\hline TVWS Database & Used & Used \\
\hline Spectrum Sensors & Used & Not Used \\
\hline
\end{tabular}

Further details of the IEEE 802.11af architecture and mechanisms are available at [8].

While both the IEEE 802.22 and IEEE 802.11af standards share many similarities such as the use of TVWS databases and geolocation services, they still have many differences. Table I summarizes such similarities and differences.

\section{IEEE 1900 STANDARD: DYNAMIC SPECTRUM ACCESS NETWORKS (DYSPAN)}

The Dynamic Spectrum Access Networks Standardization Committee (DySPAN-SC), formally known as the IEEE Standardization Coordinating Committee 41 (SCC41), targets facilitating the development of the research ideas related to distributed cognitive radio networks into standards to expedite the use of such ideas in commercial products [9]. This standardization effort is based on the IEEE P1900 initiative started in 2004. The DySPAN-SC goals are to:

- $\quad$ Standardize dynamic spectrum access radio systems and networks while focusing on improving the spectrum utilization.

- Develop new dynamic spectrum access techniques and cognitive networking methods as well as the management of radio transmission interference in such heterogenous environments.

- Coordinate the activities and the deployment of different wireless technologies to share the available spectrum and enable successful coexistence.

The DySPAN standard consists of seven Working Groups (WGs), only six of which are currently active. ${ }^{3}$ The IEEE 1900 WGs can be summarized as follows.

A. IEEE 1900.1: Definitions and Concepts for DSA: Terminology Relating to Emerging Wireless Networks, System Functionality, and Spectrum Management.

The IEEE 1900.1 standard provides the definitions and explanations of the fundamental concepts in the fields of spectrum management, cognitive radio, policy-defined radio, adaptive radio, software-defined radio, and related technologies. Such a common dictionary of terms is critical to the cognitive radio community to ensure a unified understanding of the used technical terms. The IEEE 1900.1 was approved on September 2008. In February 2011, the IEEE 1900.1

\footnotetext{
${ }^{3}$ In addition, the DySPAN-SC has created the ad hoc on Dynamic Spectrum Access in Vehicular Environments (DSA-VE) to define the DSA rules for vehicular communications.
}

Working Group has started working on a new amendment, IEEE 1900.1a. The primary goal of the IEEE 1900.1a is to incorporate the updated terms and definitions that resulted from other IEEE 1900 Working Groups into the standard. The IEEE 1900.1a amendment was published in January 2013.

B. IEEE 1900.2: Recommended Practice for the Analysis of In-Band and Adjacent Band Interference and Coexistence Between Radio Systems.

Motivated by the emergence of different spectrum management, policy-defined radio, adaptive radio, and softwaredefined radio systems, the IEEE 1900.2 WG aimed at improving the spectral efficiency and allowing the coexistence of different cognitive radio technologies. The resulting recommended practice provides the technical guidelines for analyzing the potential for the coexistence - or in contrast the interference - between radio systems operating in the same frequency band or between different frequency bands. The IEEE 1900.2 was approved on July 2008.

C. IEEE 1900.3: Recommended Practice for Conformance Evaluation of Software Defined Radio (SDR) Software Modules.

The objectives of this WG was to provide the recommended practices to help evaluating the IEEE 1900.2 output in order to assure compliance with the spectrum use requirements by using formal mathematical concepts and methods. The planned output of that standardization effort was to provide a set of tests and evaluation methods to be used in regulatory compliance testing of CR terminals. However, the IEEE P1900.3 WG was dismantled in 2008 due to the lack of volunteers.

D. IEEE 1900.4: Architectural Building Blocks Enabling Network-Device Distributed Decision Making for Optimized Radio Resource Usage in Heterogeneous Wireless Access Networks.

In February 2009, the IEEE 1900.4 standard was approved to address radio resource management and reconfiguration management in composite wireless networks comprising multiple radio access technologies. It addresses the optimization of resources in both fixed and dynamic spectrum access contexts. The IEEE 1900.4 standard defines the architectural building blocks enabling network-device distributed decision making for optimized radio resource usage in heterogeneous wireless networks. The building blocks comprising (i) network resource managers, (ii) device resource managers, and (iii) the definition of the information to be exchanged between these building blocks in heterogeneous wireless access networks for enabling coordinated network-device distributed decision making that will aid in the optimization of radio resource usage and spectrum access control. The IEEE 1900.4 defines a policybased management framework for decision making. The IEEE 1900.4 dynamic policy-based approach makes it well suited to the changing and non-homogeneous white spaces context.

The DySPAN-SC then started two work groups based on the 1900.4 standard: the IEEE 1900.4a and the IEEE 1900.4.1. The IEEE 1900.4a amendment of the original IEEE 1900.4 standard is concerned with the "architectures and interfaces for dynamic spectrum access networks in the TV white space frequency bands". The final standard would include the definition of the new devices and interfaces required for operating over the white space frequency bands in addition to IEEE 1900.4 entities and interfaces. While the white space spectrum 
might vary depending on the country or the region, the IEEE 1900.4a standard provides a common management system that is independent of the radio interface of choice. Such a unique management system enables the white spaces devices to comply with the regulation irrespective of the geographic location and time. Hence, the protection of broadcast systems will be absolutely reliable. On the other hand, the 1900.4.1 "Standard for Interfaces and Protocols Enabling Distributed Decision Making for Optimized Radio Resource Usage in Heterogeneous Wireless Networks" is responsible for providing the details of the interfaces and service access points defined in the IEEE 1900.4 standard in order to ensure interoperability between network side and terminal side components.

\section{E. IEEE 1900.5: Policy Language and Policy Architectures for Managing Cognitive Radio for Dynamic Spectrum Access Applications.}

This standard defines the policy language and the associated architecture requirements for interoperable, vendorindependent control of dynamic spectrum access functionality and behavior in radio systems and wireless networks. In other words, it defines the relationship of policy language and architecture to guarantee consistency between the regulator, the operator, the user, and the network equipment manufacturer. The work on the IEEE 1900.5 standard started in August 2008 and the draft standard was published in January of 2012. The IEEE $1900.5 \mathrm{WG}$ has recently issued two project authorization requests for extention standards: the P1900.5.1 and the P1900.5.2. The 1900.5.1 draft standard for "Policy Language for Dynamic Spectrum Access Systems" which aims at defining a vendor-independent policy language for managing the functionality and behavior of dynamic spectrum access networks based on the language requirements defined in the IEEE 1900.5 standard. Meanwhile, the P1900.5.2 "Standard Method for Modeling Spectrum Consumption Scope” defines a vendor-independent generalized method for modeling spectrum consumption of any type of use of spectrum and the attendant computations for arbitrating the compatibility among models. The P1900.5.2 authorization request was approved in March 2013. The IEEE 1900.5 WG is currently initiating the P1900.5.a as an amendment to the standard to define the detailed interfaces between policy architecture components.

\section{F. IEEE 1900.6: Spectrum Sensing Interfaces and Data Struc- tures for Dynamic Spectrum Access and other Advanced Radio Communication Systems.}

The objective of the IEEE 1900.6 WG is to define the interfaces and the data structures required to exchange sensingrelated information in order to increase the interoperability between sensors and their clients developed by different manufacturers. The logical interface and supporting data structures are defined abstractly without constraining the sensing technology, client design, or data link between the sensor and the client. It also defines the involved entities and the exchanged parameters in this process. This standard further elaborates on the service access points, service primitives, as well as the generic procedures used to realize the information exchange. The IEEE 1900.6 standard was published in April 2011. In June 2011, the IEEE 1900.6 WG started working on the IEEE 1900.6a amendment "Procedures, Protocols and Data Archive Enhanced Interfaces". The goal of this amendment is to provide the specifications that allow the integration of 1900.6-based distributed sensing systems into existing and future dynamic spectrum access networks. It enables existing legacy communication systems to benefit from adopting the IEEE 1900.6 interface as an add-on to these systems and to claim standard conformance for an implementation of the interface. Furthermore, this amendment facilitates the sharing of spectrum sensing data and other relevant data among 1900.6 based entities and external data archives. The IEEE 1900.6a amendment was completed in March 2014. Since August 2014, the WG has been working a new spectrum database amendment “ IEEE 1900.6b: Standard for Spectrum Sensing Interfaces and Data Structures for Dynamic Spectrum Access and other Advanced Radio Communication Systems".

G. IEEE 1900.7: Radio Interface for White Space Dynamic Spectrum Access Radio Systems Supporting Fixed and Mobile Operation.

This WG targets specifying a radio interface including the MAC sublayer(s) and PHY layer(s) of white space dynamic spectrum access radio systems supporting fixed and mobile operation in white space frequency bands, while avoiding causing harmful interference to incumbent users in these frequency bands. The goal is to provide means to support P1900.4a for white space management and P1900.6 to obtain and exchange sensing related information (spectrum sensing and geolocation information). The standard is not approved yet.

\section{CONCLUDING REMARKS}

In this paper, we have presented an overview of the recent and ongoing IEEE standardization efforts for CRNs. Our main focus was on both the IEEE 802.22 and IEEE 802.11af standards which exploit the superior propagation characteristics of the TV white spaces that recently emerged in the TV bands world wide. The wireless communication market already has commercial products that implement both standards. We also have discussed the IEEE 1900 DySPAN standard which aims at standardizing the definitions and key enabling techniques of CRNs as a step towards achieving coexistence and interoperability between different CRN implementations.

\section{REFERENCES}

[1] I. F. Akyildiz, W.-Y. Lee, and K. R. Chowdhury, "CRAHNs: Cognitive radio ad hoc networks," Ad Hoc Networks (Elsevier), vol. 7, no. 5, 2009.

[2] G. Gur and F. Alagoz, "Green wireless communications via cognitive dimension: an overview," IEEE Network, vol. 25, no. 2, 2011.

[3] J. Mitola III, "Cognitive radio: An integrated agent architecture for software defined radio," Ph.D. dissertation, KTH Royal Institute of Technology, 2000.

[4] IEEE 802.19 Wireless Coexistence Working Group. URL http://www.ieee802.org/19/. Last accessed Feb. 2015.

[5] IEEE Working Group on Wireless Regional Area Networks, "Enabling rural broadband wireless access using cognitive radio technology in TV whitespaces," URL http://www.ieee802.org/22/. Last accessed Feb. 2015.

[6] IEEE Std 802.22 $2^{\mathrm{TM}}$-2011, "IEEE Standard for Information Technology - Telecommunications and information exchange between systems Wireless Regional Area Networks (WRAN) - Specific requirements - Part 22: Cognitive Wireless RAN Medium Access Control (MAC) and Physical Layer (PHY) Specifications: Policies and Procedures for Operation in the TV Bands," July 2011.

[7] IEEE 802.11 Working Group, "IEEE 802.11af Draft 5.0, Amendment 5: TV White Spaces Operation," June 2013.

[8] A. B. Flores, R. E. Guerra, E. W. Knightly, P. Ecclesine, S. Pandey, "IEEE 802.11af: a standard for TV white space spectrum sharing," IEEE Communications Magazine, vol.51, no.10, pp.92-100, Oct. 2013.

[9] IEEE DySPAN Standards Committee, "Dynamic Spectrum Access Networks (DySPAN). URL http://www.dyspan-sc.org/. Last accessed Feb. 2015. 